\section{SP0156 HOW BIG DATA HELP US UNDERSTAND NEW AND OLD THERAPY TARGETS}

\section{P.E. Lipsky. Ampel Biosolutions, Charlottesville, United States}

Gene expression profiling is a valuable tool to identify the altered cellular status of cells or tissues. Metaanalysis of available gene expression profiles from numerous sources provides a means to assess the molecular alterations characteristic of autoimmune diseases, such as systemic lupus erythematosus (SLE) and to develop insights into possible new therapies. Toward this end, all of the publicly available gene expression datasets from SLE blood and tissue were assembled and a variety of analytic algorithms were employed to identify differentially expressed genes, gene modules associated with clinical features, molecular pathways associated with SLE and potential upstream regulators. This information was employed to cross-reference a variety of databases to predict novel molecular pathways and potential drug candidates. Specifially, gene expression profiles obtained from lupus affected skin, synovium and kidney were obtained, compared to metaanalyzed data obtained from active lupus B cells, $T$ cells and myeloid cells, and cross-referenced to various pathway analytic tools including Molecular Signature $\left(\mathrm{MS}^{\odot}\right)$-Scoring, Ingenuity Pathway Analysis ${ }^{\odot}$ Upstream Regulator (IPA ${ }^{\odot}-$ UR) and Library of Integrated Network Based Cellular Signatures (LINCS). More than 300 arrays from lupus patients and appropriate controls were analyzed to determine differentially expressed (DE) genes [8279 discoid skin, 5465 lupus synovium, 6381 lupus nephritis glomerulus, 5587 lupus nephritis tubulointerstitum]. Notably, the majority of lupus affected tissue DE genes were detected in more than one tissue and 439 were differentially expressed in all tissues. Tissue inflammatory/immune cell infiltration was documented by genes encoding specific cell markers as well as by unique gene expression signatures (Biologically Informed Gene Clustering, BIG-C ${ }^{\odot}$ ). Examination of curated predicted functional groups from the STRING (Search Tool for Retreaval of Interacting Genes/Proteins) output of common up-regulated transcripts in lupus tissue predicted therapeutic targets and drugs using STITCH (Search Tool for Interacting Chemicals) and IPA's BioProfiler ${ }^{\odot}$ that were confirmed by connectivity using LINCS. Analysis of gene expression datasets for the presence of specific gene modules using GSVA (Gene Set Variation Analysis) documented the overall similarity of active lupus gene sets as well as the uniqueness of individual patient samples. These results demonstrate the value of comprehensive application of orthogonal curated bioinformatics tools in identifying the role of inflammatory/immune cells in lupus pathogenesis and tissue damage. This approach demonstrated that there are molecular pathways common to all lupus patients and tissues, and there are pathways involved in inflammatory response of some but not all patients and tissues. Further analysis should generate a molecular model of lupus immunopathogenesis and could identify therapies that may be useful in all lupus patients versus those with involvement of specific tissues and treatments specifically targeted to individual lupus patients.

Grammer AC et al. Lupus (2016) 25:1150-1170.

Disclosure of Interest: None declared

DOI: 10.1136/annrheumdis-2017-eular.7234

\section{SATURDAY, 17 JUNE 2017}

\section{Optimizing treatment for osteoarthritis: take the phenotype in account "one size does not fit all"}

\section{SP0157 METABOLIC PHENOTYPE: THE TWO FACES OF OBESITY IN OSTEOARTHRITIS}

F. Berenbaum ${ }^{1,2,3} \cdot{ }^{1}$ Department of Rheumatology, Sorbonne University; ${ }^{2}$ INSERM; ${ }^{3}$ AP - HP Hospital Saint-Antoine, Paris, France

Osteoarthritis (OA) has long been considered as the consequence of tear and wear due to mechanical triggers. In that paradigm, the increased risk of OA in obese patients can be explained by overload on weight-bearing joints. The discovery of an increased risk of hand $O A$ in obese patients has changed the paradigm by opening up an additional hypothesis (not ruling out the mechanical hypothesis) based on systemic factors secreted by the adipose tissue (even the central one or/and the intraarticular one), which could have a deleterious effect on joints. More recently, cohorts data have suggested that obese patients with a metabolic syndrome (MetS) (an association of cardiometabolic factors that increase the risk of cardiovascular events i.e. diabetes, hypertension, dyslipidemia) have an increased risk of OA compared to obese patients without MetS. The most recent researches in that field highlight the role of metabolic-triggered inflammation (called meta-inflammation) on the initiation/progression of OA and the role of each of the components of the MetS independently (hyperglycemia, insulinoresistance, oxLDL, endothelial dysfunction, etc.). A better understanding of this newly defined OA phenotype, namely the MetS-associated OA, should lead to the discovery of new drugs specifically targeting this phenotype or/and to assess the efficacy of drugs in OA already on the market for diabetes, hypertension or dyslipidemia.

Disclosure of Interest: F. Berenbaum Grant/research support from: Servier, TRB Chemedica, Consultant for: Pfizer, AbbVie, GSK, Flexion Therapeutics, Nordic Bioscience, MerckSerono, Jansen, Expanscience

DOI: 10.1136/annrheumdis-2017-eular.7120

\section{SP0158 PAIN PHENOTYPE: CAN WE TAKE ADVANTAGE OF THE PLACEBO EFFECT}

A. Abhishek. Academic Rheumatology, University of Nottingham, Nottingham, United Kingdom

The discordance between pain and structural changes in osteoarthritis has been recognised for over two decades. Recent research has also identified different pain phenotypes in people with osteoarthritis. This suggests that the changes in the peripheral and central nervous system may affect both the severity and character of pain and associated features in osteoarthritis. Placebo response, the improvement in symptoms in those in the placebo arm of a randomized controlled trial, and placebo effect, the difference in improvement in those treated with placebo (in placebo arm of a randomized controlled trial) and those who have had no treatment (e.g. in the observation only arm of a three arm randomized controlled trial) are mediated by complex neural mechanisms and are especially relevant to the management of osteoarthritis. Indeed, the placebo response associated with most osteoarthritis treatments is stronger than their treatment effects. While a large placebo response is a significant obstacle to the development of new drugs and non-drug interventions for the treatment of osteoarthritis, it can be utilised as a "friend" in the day to day and long-term management of a person with osteoarthritis. This lecture will outline the pain phenotypes associated with osteoarthritis and describe the potential for placebo effect in improving osteoarthritis pain. The pain phenotypes associated with osteoarthritis that are most likely to be improved by optimizing placebo response and the underlying mechanisms will be discussed.

Disclosure of Interest: None declared

DOI: 10.1136/annrheumdis-2017-eular.7224

\section{SATURDAY, 17 JUNE 2017}

Targeting adipose tissue inflammation

\section{SP0159 ADIPOSE TISSUE - BYSTANDER OR THERAPEUTIC TARGET?}

M.I. Bokarewa. Rheumatology and Inflammation Research, University of Gothenburg, Gothenburg, Sweden

Adipose tissue (AT) is a complex endocrine organ with profound effects on body physiology. Research of recent years generated remarkable volume of information on metabolic and immune functions of AT. The lecture will focus on topics of AT biology that shed light on areas of interest linking metabolic, immune and rheumatic conditions.

AT is a recognized master regulator of energy balance and nutritional homeostasis. Cells of AT are active part in establishment of autoimmunity, and also osteoarthritis and osteoporosis. Macrophages enrich AT, change its phenotype and mediate inflammation, enhance antigen presentation. Th17 and regulatory T cells reside and operate in AT, having distinct transcriptional and functional characteristics from counterparts of the lymphoid organs. IL6 and IL33 signaling contribute to this distinction. Adipocytes communicate with other cell types by means of signal molecules adipokines, interleukins, and fatty acids and may affect blood pressure, muscle functions, wound healing, bone formation, hematopoiesis and tumor growth. Localization of AT plays important role for its properties including cellular composition, their gene expression profile and secretome, response to external forces and their therapeutic potential. Subcutaneous AT and bone marrow AT are most available and studied in humans.

Adiposity has been revealed as a risk factor for development of multiple autoimmune conditions including type 1 diabetes, rheumatoid arthritis, inflammatory bowel disease, multiple sclerosis, psoriasis and thyroiditis. It also associates with low response rate to both biologic and non-biologic anti-rheumatic treatment. Notably, cumulative evidence show a surprising protective action of AT for radiographic joint damage in arthritis.

Antirheumatic drugs have remarkable effect on the body fat composition and serum lipid profiles, and could therefore be considered as AT-targeting agents. Impact of these drugs on the composition and function of AT localized subcutaneously and in the bone marrow remains in focus of research and will provide new insights in mechanisms of insulin resistance, cardiovascular risk, bone metabolism and autoantibody production.

Production of adipokines has been proposed a mechanism through which AT fuels inflammation and severity of autoimmune diseases however direct evidence still awaits. Several adipokine targeting and adipokine stimulating therapies have been designed and experimentally tested in the models of collagen-induced arthritis, autoimmune diabetes and insulin resistance, encephalitis, and osteoporosis induced by estrogen-deficiency. In human settings, the experience of leptin substitution in anorexia neurosa and in lipodistrophy revealed positive effects on bone metabolism and improved cognition.

In conclusion, AT attracts increasing attention as an active contributor of inflammation and autoimmunity. Further studies are needed to confirm and specify the effects and pathogenic links within AT. Novel insights in mechanisms regulating the diversity of AT composition and functions may assist conversion of AT into a teammate of anti-rheumatic processes.

Disclosure of Interest: None declared

DOI: 10.1136/annrheumdis-2017-eular.7064 